\title{
Subacute necrotising encephalomyelopathy (Leigh's disease; Leigh syndrome)
}

P N Leigh, ${ }^{1,2}$ S Al-Sarraj, ${ }_{1}^{3}$ S DiMauro ${ }^{4}$

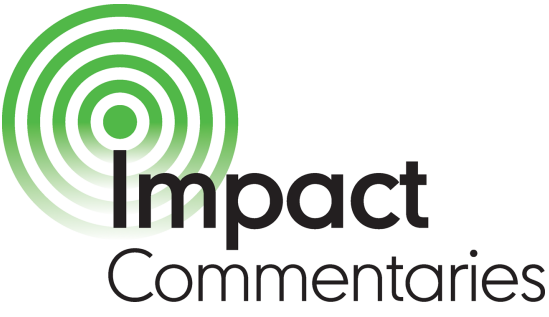

Like so many complex and incurable neurological disorders, the disease first described in this journal in 1951 by (Archibald) Denis Leigh (1915-1998; figure 1) as 'Subacute Necrotising Encephalomyelopathy' (SNE) ${ }^{1}$ remained for many years a rare and intriguing enigma, of interest mainly to paediatric neurologists and neuropathologists. The more recent history of this disorder, now designated Leigh syndrome (LS) in view of its heterogeneity, exemplifies the revolution in clinical neurosciences, culminating in the understanding of the consequences of multiple mitochondrial defects through fundamental insights derived from molecular genetics, and attributing LS to a tale of two genomes, that is, to mutations in the mitochondrial DNA (mtDNA) or to nuclear DNA. $^{2}$

The patient described by Leigh was an infant who had died aged 7 months in King's College Hospital, London, following a brief encephalopathic illness marked by drowsiness, respiratory difficulties, blindness, deafness and bilateral spasticity. The neuropathological features of the condition recognised as distinctive by Leigh included a strikingly symmetrical proliferation of smaller blood vessels, neuronal degeneration and gliosis targeting particularly parts of the thalamus, midbrain, pons, medulla and dorsal spinal cord.

${ }^{1}$ Brighton and Sussex Medical School, Trafford Centre for Biomedical Science, University of Sussex, East Sussex, UK; ${ }^{2}$ Department of Basic and Clinical Neuroscience, King's College London, London, UK; ${ }^{3}$ Department of Neuropathology, King's College Hospital NHS Foundation Trust, London, UK; ${ }^{4}$ Department of Neurology, Columbia University Medical Center, New York, USA

Correspondence to Professor P N Leigh, Brighton and Sussex Medical School, Trafford Centre for Biomedical Science, University of Sussex, East Sussex BN1 9RY, UK; p.leigh@bsms.ac.uk
Subacute necrotising encephalopathy in an infant.

Author: A Denis Leigh

Published: I Neurol Neurosurg

Psychiatry 1951;14:216-21.

Citations: 649

There was loss of myelin in the optic nerves and in association with the necrotising lesions in the grey matter. Leigh recognised striking similarities—but also important differences-between the pathological lesions seen in the infant with SNE and in Wernicke's encephalopathy. In the former, the subthalamic nuclei and mammillary bodies were strikingly spared, in contrast to Wernicke's encephalopathy.
$\mathrm{He}$ speculated that the lesions in SNE might also be attributable to a nutritional deficiency, a theme to which he and others returned some years later, hoping that treatment with thiamine or related agents might lie in that direction. Although most patients with LS do not convincingly respond to such treatment, biotin and thiamine may dramatically improve the condition of biotin-responsive basal ganglia disease, a disorder that can mimic LS. $^{3}$ Nonetheless, the notion that a metabolic defect might be at the heart of the problem, and that metabolic stress might precipitate neuronal damage ${ }^{4}$ has been amply borne out.

Leigh's career exemplifies a lost interdisciplinary flexibility. After qualifying in medicine in Manchester in 1938, he trained with the eminent neurosurgeon Sir Jeffrey Jefferson, intending a career in neurology or neurosurgery. However, at the outbreak of war he joined the Royal Army Medical Corps, and after a spell as a regimental medical officer, was posted (through the influence of Jefferson) to the Oxford Head Injuries Unit where he worked with the outstanding neurological leaders and

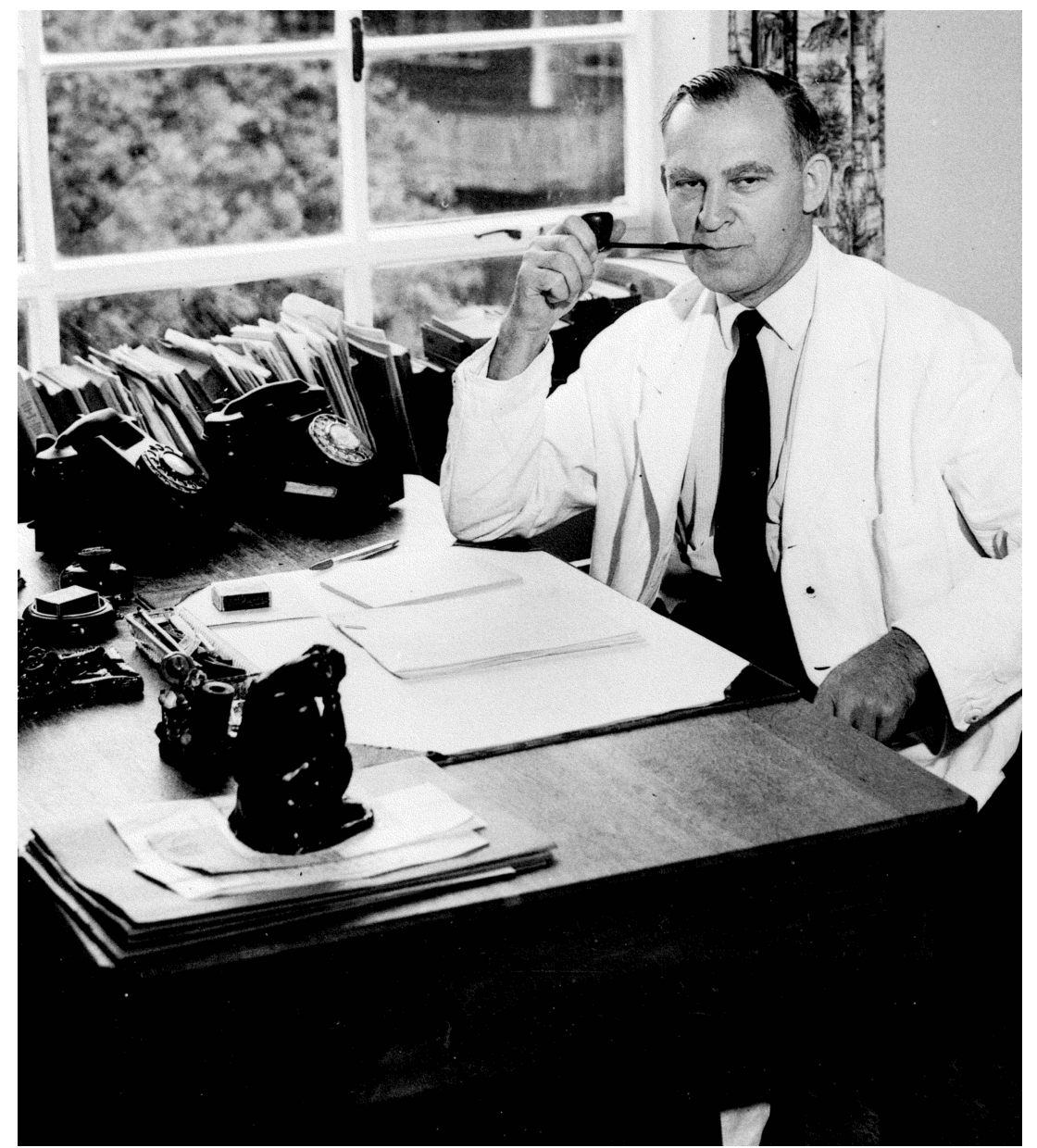

Figure 1 Dr A Denis Leigh in his office at the Maudsley Hospital, London, circa 1966. 
aspirants of that generation. From 1942 he served in India and Burma, ending the war as Lieutenant-Colonel and Advisor in Neurology to the Eastern army, while undertaking research (supervised by Derek Denny-Brown) into diphtheritic polyneuropathy and the neurology of nutritional disorders in prisoners of war.

He returned home as First Assistant (Senior Resident) to the Neurology Department at The London Hospital, working for Sir (later Lord) Russell Brain and George Riddoch, and in 1948 he was awarded a Nuffield Fellowship to study at the Massachusetts General Hospital in Boston where, influenced by the neuropsychiatrist Stanley Cobb, he decided to switch to a career in psychiatry. He felt that psychiatry offered greater challenges and more exciting prospects for therapy than neurology at that time.

On his return to London in 1949, while waiting for a post in psychiatry at the Maudsley Hospital, he was attached to the Department of Neuropathology under the benevolent supervision of Professor Alfred Meyer (1895-1990). ${ }^{5}$ Meyer asked Leigh to study the brain of this child, and thus chance, and a generous mentor, led Leigh to identify a previously unknown neurological disease now recognised as the most common mitochondrial disorder of childhood, affecting 1 in 40000 of newborn children in the USA. ${ }^{6}$

Following this productive neuropathological interlude, Leigh pursued a career as a general psychiatrist with an interest in psychosomatic disorders, founding the Journal of Psychosomatic Research, of which he was Editor-in-Chief for many years, and becoming Secretary-General of the World Psychiatric Association (1966-1978). Nonetheless, he maintained his interest in SNE throughout his life, painstakingly collecting reprints and photocopies. Naturally, he was proud of his role in identifying the disease bearing his name. As David Marsden, a colleague at the Maudsley and Institute of Psychiatry, remarked-not many clinicians make significant contributions across professional disciplines in this way.

Intrigued by the claim in 1969 that the urine of patients with SNE contained an inhibitor of thiamine phosphotransferase, Leigh returned briefly to the study of LS in collaboration with Henry McIlwain at the Institute of Psychiatry. They hypothesised that patients with LS might have defects in the thiamine metabolism, and measured the activity of erythrocyte transketolase (ETK) in samples from over 100 patients with pathologically proven or suspected SNE. It turned out that the urine inhibitor was not a reliable test for SNE and there was no indication that ETK activity was abnormal in SNE. ${ }^{7}$

The first indications that LS might be a mitochondrial disorder can be traced to1965 with the identification of lactic acidosis in a child with intermittent attacks of vomiting and fluctuating failure to thrive. The observation in 1968 of a deficiency of liver pyruvate decarboxylase in a patient with Leigh's disease led to other defects in pyruvate metabolism being detected and in 1977 cytochrome C oxidase deficiency was identified in muscle of a patient with SNE (for a comprehensive review of these developments and of LS generally, see Baertling et al, 2013). ${ }^{8}$

The two decades following Leigh's paper saw the clinical phenotypes associated with LS expand to include rare patients with adolescent and adult onset, and a wide variety of rapidly progressive or chronic presentations. The range of biochemical abnormalities associated with what was evidently a syndrome rather than a single disease entity widened. It became clear that this was a genetically determined disorder that could be inherited as an autosomal recessive trait, but which might also be maternally inherited (table 1).

LS can now be suspected and diagnosed in life through the biochemical profile of lactic acidaemia, changes in blood and urine aminoacids and organic acids, muscle pathology, analysis of oxidative phosphorylation deficits in muscle and fibroblast cultures, MRI (and MR spectroscopic) changes, but most accurately and specifically through genetic analysis. ${ }^{2} 8$ However, the clinical, biochemical, and genetic spectrum of LS is complex, and over 60 entries are listed in Online Mendelian Inheritance in Man (OMIM) (http://www.omim.org). Sequencing of the mitochondrial genome is feasible, but to date a candidate gene approach has been necessary to detect nuclear gene mutations. However, whole exome sequencing should transform the detection of relevant mutations in LS. 9

On the basis of current knowledge, mutations in several mtDNA protein-coding genes most notably affecting subunits of complex I (MTND5, MTND3) or complex V (MTATP6) cause maternally inherited LS (MILS), manifesting differently severe symptoms in maternal relatives (neuropathy, ataxia and retinitis pigmentosa NARP) according to the degree of heteroplasmy. Mendelian heredity of LS started with the recognition of mutations in one subunit of complex II, but mutations in many subunits of the giant complex I followed the example of 'direct hits', that is
Table 1 Distribution of genetic lesions in components of the respiratory chain

\begin{tabular}{lll}
\hline \multicolumn{2}{l}{ Causes of Leigh syndrome } \\
\hline Defect & Transmission & Frequency \\
\hline Complex I & AR, M & ++++ \\
Complex II & AR & + \\
Complex IV & AR & +++ \\
Complex V & M, AR & ++ \\
tRNA $^{\text {Leu(UUR) }}$ & M & + \\
tRNA $^{\text {Lys }}$ & M & + \\
CoQ10 & AR & + \\
PDHC & XR, AR & +++ \\
\hline
\end{tabular}

In Leigh's syndrome. Inheritance: $A R$, autosomal recessive; CoQ10, coenzyme Q10; $M$, maternal; PDHC, pyruvate dehydrogenase complex; XR, X-liked recessive.

mutations, affecting directly subunits of respiratory chain complexes. More common are LS's due to 'indirect hits', that is, to mutations affecting assembly proteins needed to put together mtDNA-encoded and nDNA-encoded subunits, most notably involving assembly proteins for complex IV (cytochrome c oxidase), complex V (ATP synthase), or biosynthetic enzymes for coenzyme Q10 (CoQ10). ${ }^{2}$ Finally, defects involving many steps of mtDNA translation can present with LS, and, more rarely, LS can be part of the clinical presentations due to defects in the lipid milieu of the inner mitochondrial membrane, and to defects in mtDNA maintenance.

In summary, LS can be inherited by maternal inheritance or, much more commonly, by autosomal recessive inheritance. However, there are a few examples of $\mathrm{X}$ linked transmission, due to one respiratory chain defect (complex I) or to disorders of pyruvate metabolism. The stereotypical lesions of LS reflect the ravages caused by defective oxidative metabolism on the developing nervous system.

We can only speculate on the genetic lesion in Leigh's patient. However, a few of the original celloidin-embedded slides came to light following Leigh's death in 1998. They were stored in an unlabelled tin found in a cupboard in the family home. Sadly, no trace of the tissue blocks or other material has been found and attempts to obtain DNA from some of the celloidin sections have so far failed (DiMauro, personal communication). The remaining sections are now preserved in the Department of Neuropathology at King's College Hospital.

Many therapies have been suggested to correct metabolic changes detected in LS but none have been effective. Perhaps a new era of experimental therapeutics will 
open with the creation of animal models, such as the Ndufs 4 knockout mouse. $N d u f_{s} 4$ gene mutations have been identified in LS. This gene encodes $\mathrm{NADH}$ dehydrogenase (ubiquinone) Fe-S protein 4 , a protein concerned with the integrity of mitochondrial complex $1 .^{10}$ Mice lacking this protein manifest clinical and pathological features closely similar to the human disease. Rapamycin, which inhibits the mammalian target of rapamycin (mTOR) improved survival and slowed disease progression, perhaps by moving away from glycolysis towards amino acid catabolism. A hopeful prospect for some individuals and families with mtDNA lesions could be mitochondrial replacement. $^{11}$

Throughout his career Leigh corresponded compassionately with families whose children had been afflicted by 'his' disease. He always hoped to see an effective treatment introduced. Although the complexity of the condition warns against simple solutions, the prospects for effective treatments of Leigh's syndrome-and other mitochondrial disorders-may at last be brightening.
Correction notice This paper has been corrected since it was published online first. The citation box on the previous page has been updated.

Competing interests PN Leigh is the son of $A D$ Leigh. Provenance and peer review Commissioned; internally peer reviewed.

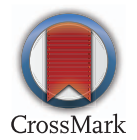

To cite Leigh PN, Al-Sarraj S, DiMauro S. J Neurol Neurosurg Psychiatry 2015;86:363-365.

Received 26 November 2014

Accepted 14 December 2014

Published Online First 13 January 2015

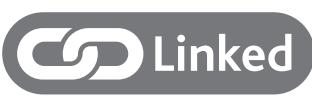

http://dx.doi.org/10.1136/jnnp-2012-304426

J Neurol Neurosurg Psychiatry 2015;86:363-365. doi:10.1136/jnnp-2012-304601

\section{REFERENCES}

1 Leigh D. Subacute necrotising encephalopathy in an infant. J Neurol Neurosurg Psychiatry 1951;14:216-21.

2 DiMauro S, Schon EA, Carelli V, et al. The clinical maze of mitochondrial neurology. Nat Rev Neurol 2013;9:429-44.
3 Tabarki B, Al-Shafi S, Al-Shahwan S, et al. Biotin-responsive basal ganglia disease revisited: clinical radiologic, and genetic findings. Neurology 2013:80:261-7.

4 Cavanagh JB, Harding BN. Pathogenic factors underlying the lesions in Leigh's disease. Tissue responses to cellular energy deprivation and their clinico-pathological consequences. Brain 1994;117:1357-76.

5 Cavanagh JB. Alfred Meyer. Neuropathol Appl Neurobiol 1991;17:83-7.

6 Darin N, Oldfors A, Moslemi AR, et al. The incidence of mitochondrial encephalomyopathies in childhood: clinical features and morphological, biochemical, and DNA abnormalities. Ann Neurol 2001;49: 377-83.

7 McBurney A, Leigh D, Mcllwain H. Erythrocyte transketolase activity in suspected cases of Leigh's disease, or subacute necrotising encephalomyelopathy. Arch Dis Child 1980;55:789-94.

8 Baertling F, Rodenburg RJ, Schaper J, et al. A guide to diagnosis and treatment of Leigh syndrome. I Neurol Neurosurg Psychiatry 2014;85:257-65.

9 Taylor RW, Pyle A, Griffin H, et al. Use of whole-exome sequencing to determine the genetic basis of multiple mitochondrial respiratory chain complex deficiencies. JAMA 2014;312:68-77.

10 Johnson SC, Yanos ME, Kayser EB, et al. mTOR inhibition alleviates mitochondrial disease in a mouse model of Leigh syndrome. Science 2013;342: $1524-8$.

11 Chinnery PF, Craven L, Mitalipov S, et al. The challenges of mitochondrial replacement. PLoS Genet 2014; 10:e1004315. 